\title{
Enhancement of equivalence class formation by pretraining discriminative functions
}

\author{
Richard K. Nartey • Erik Arntzen • Lanny Fields
}

Published online: 26 November 2014

(C) Psychonomic Society, Inc. 2014

\begin{abstract}
The present experiment showed that a simple discriminative function acquired by an abstract stimulus through simultaneous and/or successive discrimination training enhanced the formation of an equivalence class of which that stimulus was a member. College students attempted to form three equivalence classes composed of three nodes and five members $(\mathrm{A} \rightarrow \mathrm{B} \rightarrow \mathrm{C} \rightarrow \mathrm{D} \rightarrow \mathrm{E})$, using the simultaneous protocol. In the PIC group, the $\mathrm{C}$ stimuli were pictures and the A, B, $\mathrm{D}$, and $\mathrm{E}$ stimuli were abstract shapes. In the $\mathrm{ABS}$ group, all of the stimuli were abstract shapes. In the SIM + SUCC (simultaneous and successive) group, simple discriminations were formed with the $\mathrm{C}$ stimuli through both simultaneous and successive discrimination training before class formation. Finally, in the SIM-only and SUCC-only groups, prior to class formation, simple discriminations were established for the $\mathrm{C}$ stimuli with a simultaneous procedure and a successive procedure, respectively. Equivalence classes were formed by $80 \%$ and $70 \%$ of the participants in the PIC and SIM + SUCC groups respectively, by $30 \%$ in the SUCC-only group, and by $10 \%$ apiece in the ABS and SIM-only groups. Thus, pretraining of combined simultaneous and successive discriminations enhanced class formation, as did the inclusion of a meaningful stimulus in a class. The isolated effect of forming successive discriminations was more influential than that of forming simultaneous discriminations. The establishment of both discriminations together produced an enhancement greater than the sum of the two procedures alone. Finally, a
\end{abstract}

R. K. Nartey $(\bowtie) \cdot$ E. Arntzen $(\bowtie)$

Department of Behavioral Science, Oslo and Akershus University

College, PO Box 4 St. Olavs Plass, 0130 Oslo, Norway

e-mail: rknartey@yahoo.co.uk

e-mail: erik.arntzen@equivalence.net

L. Fields

Queens College and The Graduate School, City University of New

York, New York, NY, USA sorting test documented the maintenance of the classes formed during the simultaneous protocol. These results also provide a stimulus control-function account of the class-enhancing effects of meaningful stimuli.

Keywords Meaningfulness · Stimulus equivalence · Simultaneous discrimination $\cdot$ Successive discrimination . Pictures $\cdot$ Sorting tests $\cdot$ College students

When the relations among a finite set of perceptually disparate stimuli can show the properties of reflexivity, symmetry, and transitivity, alone and in combination, those stimuli are said to be equivalent to each other and to be acting as members of an equivalence class (Sidman \& Tailby, 1982). These stimuli then can be used substitutably or interchangeably with each other. For instance, if the stimuli are represented by the letters $\mathrm{A}, \mathrm{B}, \mathrm{D}, \mathrm{C}$, and $\mathrm{E}$, after the training of the conditional relations $\mathrm{AB}, \mathrm{BC}, \mathrm{CD}$, and $\mathrm{DE}$, their functionality as members of an equivalence class would be documented by the emergence of untrained derived reflexive relations $(\mathrm{AA}, \mathrm{BB}, \mathrm{CC}, \mathrm{DD}$, and $\mathrm{EE})$, symmetrical relations (BA, $\mathrm{CB}, \mathrm{DC}$, and $\mathrm{ED})$, transitive relations $(\mathrm{AC}, \mathrm{AD}, \mathrm{AE}, \mathrm{BD}, \mathrm{BE}$, and $\mathrm{CE})$, and equivalence relations (CA, DA, EA, DB, EB, and EC) (Fields \& Verhave, 1987; Sidman, 1994; Sidman \& Tailby, 1982). When such a class is formed, the stimuli can be substituted for each other in new settings, and typically a response trained to one stimulus will also be evoked by the other stimuli in the class without benefit of additional direct training; the class then acts as a response transfer network.

In basic research settings, it is usual to establish classes amongst stimuli in set of nominally meaningless stimuli. When attempts to form classes are made using the simultaneous protocol, which involves first training all baseline relations and then testing all derived relations together with the baseline relations, typically classes are quite unlikely to be 
formed. On the other hand, the inclusion of a meaningful stimulus in a set of meaningless stimuli enhances the likelihood of class formation (Arntzen, 2004; Arntzen \& Lian, 2010; Fields, Arntzen, Nartey, \& Eilifsen, 2012; Nartey, Arntzen, \& Fields, 2014), whether the meaningful stimulus was used as a node (Arntzen \& Lian, 2010; Fields et al., 2012) or a single stimulus (Arntzen, 2004; Nartey et al., 2014).

Meaningful stimuli, however, can serve at least two discriminative functions: that of a simple successive discrimination and/or that of a simple simultaneous discrimination (Fields et al., 2012; Travis, Fields, \& Arntzen, 2014) . Thus, the class-enhancing effect of a meaningful stimulus could be attributed to either or both of its presumed discriminative functions. Fields et al. (2012) showed that the formation of an equivalence class (ABCDE) that consisted of meaningless stimuli was enhanced by the pre-class-formation establishment of both discriminative functions with an abstract $\mathrm{C}$ stimulus. This finding supported the view that the classenhancing effects of a meaningful stimulus can be accounted for in part by the discriminative functions served by meaningful stimuli.

As we noted above, a simple discrimination can be established on a successive or a simultaneous basis. In a simple successive discrimination, the discriminative stimulus $\left(\mathrm{S}^{\mathrm{D}}\right)$ and $\mathrm{S}-d e l t a\left(\mathrm{~S}^{\Delta}\right)$ are presented separately, and a reinforcer is presented only if a particular response occurs in the presence of the $\mathrm{S}^{\mathrm{D}}$. Such a discrimination is formed when the response comes to occur more often in the presence of the $\mathrm{S}^{\mathrm{D}}$ than in the presence of the $\mathrm{S}^{\Delta}$ (Mcllvane, 2013; Saunders \& Green, 1999). In a simultaneous discrimination, the $\mathrm{S}^{\mathrm{D}}$ and $\mathrm{S}^{\Delta}$ are presented concurrently with each trial, and reinforcement is presented for selection of the $S^{D}$. Such a discrimination is formed when the $S^{D}$ is selected on more trials than the $\mathrm{S}^{\Delta}$ (Mcllvane, 2013; Saunders \& Green, 1999).

Fields et al. (2012) studied how the combined acquisition of simultaneous (SIM) and successive (SUCC) discriminative functions by an abstract stimulus prior to equivalence class formation enhanced the formation of classes that included these stimuli. When the A-E stimuli were all abstract shapes, none of the participants formed classes. However, when the A, B, D, and E stimuli were abstract shapes and the $\mathrm{C}$ stimuli were meaningful pictures, $80 \%$ of the participants formed classes. When the A-E stimuli were abstract shapes and the C stimuli became $\mathrm{S}^{\mathrm{D}} \mathrm{S}$ through the training of both simultaneous and successive discriminations prior to class formation, $50 \%$ of participants formed classes. That experiment, however, did not identify the class-enhancing effects of the pre-classformation establishment of the successive and simultaneous discriminations in isolation. Travis et al. (2014) extended that analysis by exploring the effects of some, but not all, of those possibilities. In that study, they assessed the effects of SIM + SUCC discrimination training and of SIM-only training, but not the effects of SUCC-only training.
The present experiment explored all of those possibilities. Participants attempted to form three 3-node, 5-member equivalence classes by the sequential training of $\mathrm{AB}, \mathrm{BC}, \mathrm{CD}$, and $\mathrm{DE}$ relations, after which the emergence of the classes was assessed with a test that involved the presentation of all emergent-relation probes in the same test block. Five different pre-class-formation discrimination training groups were studied, with the first three being direct replications of the groups explored by Fields et al. (2012). In the ABS group, the A-E stimuli represented abstract shapes that were difficult to name, and thus, nominally meaningless. In the meaningful stimulus (PIC) group, the $\mathrm{C}$ stimuli were nameable and meaningful pictures, whereas the $\mathrm{A}, \mathrm{B}, \mathrm{D}$, and $\mathrm{E}$ stimuli were the same abstract shapes used in the ABS group. In the SIM + SUCC group, the $\mathrm{C}$ stimuli were established as $\mathrm{S}^{\mathrm{D}} \mathrm{s}$ by using both simultaneous and successive discrimination training prior to class formation. The present experiment also isolated the class-enhancing effects of successive discrimination training alone in a SUCC-only group, and of simultaneous discrimination training alone in a SIM-only group. A comparison of the likelihoods of class formation in each of these groups indicated that SIM and SUCC discrimination training, either alone or in combination, enhanced the formation of equivalence classes. These results also indicate that the discriminative functions presumably served by meaningful stimuli could account for their enhancement of equivalence class formation.

\section{Method}

\section{Participants}

Fifty university students ( 25 males and 25 females) between the ages of 19 and 23 years participated voluntarily in this study $(M=21$ years, $S D=1.15)$. The participants had no prior knowledge of stimulus equivalence research and methodology. Four other participants who started the experiment either quit or were dismissed because they did not acquire the baseline relations after $2 \mathrm{~h}$ of training, and new participants were recruited to replace them. Participants were assigned on a block-randomized basis to one of five experimental groups, and experimental sessions were run individually.

\section{Apparatus}

Setting The experiments were conducted in the graduate seminar room of the Department of Psychology at the University of Ghana, Legon, which measured approximately $5 \mathrm{~m}$ square and was furnished with tables and chairs.

Hardware An HP Compaq nc6320 laptop computer with an 1828-MHz Intel Centrino processor and a screen with a 16.8in. diagonal length and $16 \times 9$ horizontal-to-vertical ratio were 
used to conduct the experiments. An external mouse was used by participants to control the position of the cursor throughout the experiment.

Software A software program made by Psych Fusion Software in collaboration with the second author was used in the training and testing of all conditional discriminations for all of the participants. The software controlled the presentation of all stimuli and also made recordings of data, including the trial number, number of training trials, reaction time to the sample and comparison stimuli, whether participants made the correct or incorrect comparison choice, and whether or not programmed consequences were delivered. This software provided a summary of symmetry and of all derived-relation tests, as well as the duration of the experiment.

Stimuli Figure 1 shows the stimulus sets used in the experiment. In the top two sections of the figure are the stimuli- 15 abstract stimuli and the three familiar picture stimuli used as members of the equivalence classes - whereas the bottom section shows the stimuli used during discrimination training. The abstract stimuli were displayed in black and the picture stimuli in color, both on a white background. Small plastic-laminated pictures $3.8 \mathrm{~cm}$ square in size were made from the 15 abstract stimuli and the pictures to be used in the experiment. The size of the touch-sensitive areas on the screen was $9.4 \times 3.4 \mathrm{~cm}$.

\begin{tabular}{|c|c|c|c|}
\hline & 1 & 2 & 3 \\
\hline A & $\Pi$ & \% & $\beta$ \\
\hline B & $y$ & 邓 & $\vec{P}$ \\
\hline C & ๆ & $\beta$ & 7 \\
\hline D & 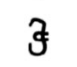 & ל & 8 \\
\hline $\mathrm{E}$ & $\bar{X}$ & 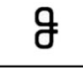 & $\hat{\vartheta}$ \\
\hline Pictures & 迹 & [2ag & (20) \\
\hline c & 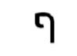 & $\beta$ & 7 \\
\hline $\mathrm{P}$ & $\overline{1}$ & $x$ & $\breve{n}$ \\
\hline R & $\lambda$ & $P$ & $ל$ \\
\hline $\mathrm{s}$ & ظ & ll & $\Phi$ \\
\hline$x$ & ラ & ب ب & $N$ \\
\hline Y & r & ك & s \\
\hline z & 7 & $\Sigma$ & $\omega$ \\
\hline
\end{tabular}

Fig. 1 The stimuli used as members of the equivalence classes were the abstract and familiar picture stimuli shown in the two top sections. The bottom section shows the abstract stimuli used during simple discrimination training
Procedure

Design The participants were assigned on a blockrandomized basis to one of five groups: (1) abstract $\mathrm{C}$ stimulus (ABS), (2) meaningful C stimulus (PIC), (3) abstract C pretrained with both simultaneous and successive discrimination (SIM + SUCC), (4) abstract C pretrained with simultaneous discrimination only (SIM-only), and (5) abstract C pretrained with successive discrimination only (SUCC-only.)

Informed consent All participants were asked to take a seat and given an informed consent document to read upon arrival at the experimental setting. The consent document informed participants that they were about to participate in an experiment in the field of behavior analysis, and that it will last approximately one-and-a-half hours. They also read that they were required to respond to certain stimuli on the screen of a computer with mouse clicks and that there were no known harmful effects of participating in the study. Participants were also informed that they were free to withdraw from the experiment at any time without any negative consequences. After reading, those who agreed participate signed the forms and began the experiment.

Categorization test: Card sorting After signing the informed consent document, the participants remained seated in the experimental cubicle, were given 15 plastic-laminated cards that corresponded to the stimuli to be used in the group to which each participant had been assigned, and were told to "put the cards into groups." Participants in the ABS, SIM + SUCC, SIM-only, and SUCC-only groups were presented with 15 abstract stimulus cards, whereas those in the PIC group were given 12 abstract stimuli and the three picture $\mathrm{C}$ stimuli. They were asked to "put the cards into groups" again after the experiment.

Instructions Participants remained seated behind the computer and were presented with the following instructions on the computer screen:

In a moment a stimulus will appear in the middle of the screen. Click on this by using the computer mouse. Three stimuli will then appear in three corners of the screen. Choose one of them by clicking on it with the mouse. If you choose the stimulus we have defined as correct, words like "very good," "excellent," and so on will appear on the screen. If you press a wrong stimulus, the word "wrong" will appear on the screen. At the bottom of the screen, the number of correct responses you have made will be counted. During some stages of the experiment, the computer will NOT tell you if your choices are correct or wrong. Please do your best to get everything right. Thank you and good luck! 
No further instructions were given before or after the experiment started.

Trial structure and contingencies All participants were exposed to the simultaneous protocol to form equivalence classes. Some of them had had some simple discrimination training prior to that. In all cases, however, all training and testing were done using trials presented in a matching-to-sample format that had the following parameters.

Each trial began with the presentation of the sample stimulus in the middle of the screen. Clicking on the sample stimulus with the computer mouse immediately resulted in the presentation of the three comparison stimuli in three of the corners of the screen, while the sample stimulus still remained on the screen. A comparison stimulus was selected by moving the mouse cursor to it and pressing the left button on the mouse. Correct responses, in the form of choosing the comparison stimulus according to the experimenter-designated classes, resulted in the removal of the sample and comparisons stimuli and the presentation of the word correct, very good, super, or excellent on the screen. Any other response produced the word wrong on the screen. If a programmed consequence was presented after the selection of a comparison, it was displayed in the middle of the screen for $1,000 \mathrm{~ms}$. Termination of the programmed consequences message was followed by a $500-\mathrm{ms}$ intertrial interval. Between trials, the mouse cursor was returned to the center of the screen.

Equivalence class formation The simultaneous protocol was used to establish equivalence classes through a three-stage process, each of which consisted of blocks of trials. First, the baseline relations for the equivalence classes were trained in a serialized manner until the achievement of a mastery criterion. Second, the baseline relations were maintained in the presence of blocks that contained decreasing proportions of reinforced trials. Third, all of the baseline relations, as well as all of the derived symmetrical, transitive, and equivalence relation probes, were presented randomly in one emergent-relations test block.

Acquisition of baseline relations The baseline relations were trained in five serialized phases, with programmed consequences provided for the selection of comparisons for each trial. Phase 1 was for the training of $\mathrm{AB}$ relations in a block containing nine trials - three from each of the classes. A mastery criterion of at least $90 \%$ correct was required to demonstrate acquisition of the relations. Participants repeated each block until the mastery criterion was met. Phases 2,3 , and 4 were the same as Phase 1 , except that the $\mathrm{BC}, \mathrm{CD}$, and $\mathrm{DE}$ relations were trained in each phase, respectively. An equalization block was used to ensure that each of the baseline relations was trained the same number of times.
Phase 5 involved the inclusion of all the relations trained in the first four blocks: $\mathrm{AB}+\mathrm{BC}+\mathrm{CD}+\mathrm{DE}$. The trials presented in the Phase 5 blocks were A1/B1B2B3, A2/ B1B2B3, A3/B1B2B3, B1/C1C2C3, B2/C1C2C3, B3/ C1C2C3, C1/D1D2D3, C2/D1D2D3, C3/D1D2D3, D1/ E1E2E3, D2/E1E2E3, and D3/E1E2E3. For each of the trial representations, the first stimulus was the sample, and the other three were the comparison stimuli, where the comparison in bold text above was the correct comparison. Therefore, in each of the trials, participants were expected to match the samples to the correct comparisons. The comparison stimuli were presented in different positions in the three corners of the screen on a randomized basis. The block contained 36 trials (three presentations of each of the 12 trial types listed above). The block was repeated until correct comparisons were selected on at least $90 \%$ of the trials of each baseline relation in it; the achievement of this mastery criterion defined the acquisition of the baseline relations.

Maintenance of baseline trials In this phase, participants continued with the training blocks, but with reduced programmed consequences across blocks. After the last block of acquisition, the percentages of trials in a block that produced the programmed consequences were reduced to $75 \%, 50 \%, 25 \%$, and $0 \%$, in that order. The mastery criterion for each block was the selection of correct comparisons on at least $90 \%$ of the trials (33 of 36) of each baseline relation in a block. This programmed reduction of consequences density was conducted to ensure that the baseline relations would be intact even without reinforcement and that the maintenance of baseline relations would not be discriminable from the subsequently presented test blocks, which were also administered without programmed consequences. For each level of programmed consequences, the trials that produced the programmed consequences were randomized in a block. The maintenance phase was completed with the mastery level of responding in the last block of 36 baseline trials with no programmed consequences. Table 1 shows a full overview of each of the experimental phases.

Emergent-relations tests The last baseline block, which was presented with no consequences, was followed by an emergent-relations test block that contained 180 trials made up of 36 baseline trials, 36 symmetry trials, 54 one-node trials, 36 two-node trials, and 18 three-node trials. All of these trials were randomly presented and without programmed consequences.

Equivalence classes could emerge on either an immediate or a delayed basis. To provide for the measurement of delayed emergence, the emergent-relations test block was divided into two halves of 90 trials each. The first and second sets were referred to as Test Blocks 1 and 2, respectively. The formation of equivalence classes was defined by the selection of 
Table 1 Training and testing sequences

\begin{tabular}{|c|c|c|c|c|}
\hline Experimental Phases & Trial Types & $\begin{array}{l}\text { Programmed } \\
\text { Consequences (\%) }\end{array}$ & $\begin{array}{l}\text { Min. \# of } \\
\text { Trials }\end{array}$ & Criterion \\
\hline \multicolumn{5}{|l|}{ Acquisition of Baseline Trials } \\
\hline 1. Serialized trials & $\mathrm{A} 1 \mathrm{~B} 1, \mathrm{~A} 2 \mathrm{~B} 2, \mathrm{~A} 3 \mathrm{~B} 3$ & 100 & 9 & 9 \\
\hline 2. Serialized trials & $\mathrm{B} 1 \mathrm{C} 1, \mathrm{~B} 2 \mathrm{C} 2, \mathrm{~B} 3 \mathrm{C} 3$ & 100 & 9 & 9 \\
\hline 3. Serialized trials & $\mathrm{C} 1 \mathrm{D} 1, \mathrm{C} 2 \mathrm{D} 2, \mathrm{C} 3 \mathrm{D} 3$ & 100 & 9 & 9 \\
\hline 4. Serialized trials & D1E1, D2E2, D3E3 & 100 & 9 & 9 \\
\hline $\begin{array}{l}\text { 5. Mixed trials } \\
\text { (trials presented randomly) }\end{array}$ & $\begin{array}{l}\text { A1B1, A2B2, A3B3, B1C1, B2C2, B3C3 C1D1, C2D2, } \\
\text { C3D3, D1E1, D2E2, D3E3 }\end{array}$ & 100 & 36 & 34 \\
\hline \multicolumn{5}{|c|}{ Maintenance: Fading of Programmed Feedback } \\
\hline $\begin{array}{l}\text { 6. Mixed trials (trials presented } \\
\text { randomly) }\end{array}$ & $\begin{array}{l}\text { A1B1, A2B2, A3B3, B1C1, B2C2, B3C3 C1D1, C2D2, } \\
\text { C3D3, D1E1, D2E2, D3E3 }\end{array}$ & 75 & 36 & 34 \\
\hline $\begin{array}{l}\text { 7. Mixed trials (trials presented } \\
\text { randomly) }\end{array}$ & $\begin{array}{l}\text { A1B1, A2B2, A3B3, B1C1, B2C2, B3C3 C1D1, C2D2, } \\
\text { C3D3, D1E1, D2E2, D3E3 }\end{array}$ & 50 & 36 & 34 \\
\hline $\begin{array}{l}\text { 8. Mixed trials (trials presented } \\
\text { randomly) }\end{array}$ & $\begin{array}{l}\text { A1B1, A2B2, A3B3, B1C1, B2C2, B3C3 C1D1, C2D2, } \\
\text { C3D3, D1E1, D2E2, D3E3 }\end{array}$ & 25 & 36 & 34 \\
\hline $\begin{array}{l}\text { 9. Mixed trials (trials presented } \\
\text { randomly) }\end{array}$ & $\begin{array}{l}\text { A1B1, A2B2, A3B3, B1C1, B2C2, B3C3 C1D1, C2D2, } \\
\text { C3D3, D1E1, D2E2, D3E3 }\end{array}$ & 0 & 36 & 34 \\
\hline \multicolumn{5}{|l|}{$\begin{array}{l}\text { Test for Derived Relations } \\
\text { All trial types randomly intermixed }\end{array}$} \\
\hline \multicolumn{5}{|c|}{ Baseline trials } \\
\hline & $\begin{array}{l}\text { A1B1, A2B2, A3B3, B1C1, B2C2, B3C3 C1D1, C2D2, } \\
\text { C3D3, D1E1, D2E2, D3E3 } \\
\text { Symmetry trials }\end{array}$ & 0 & 36 & 34 \\
\hline & $\begin{array}{l}\text { B1A1, B2A2, B3A3, C1B1, C2B2, C3B3 D1C1, D2C2, } \\
\text { D3C3, E1D1, E2D2, E3D3 } \\
\text { One-node trials }\end{array}$ & 0 & 36 & 34 \\
\hline & $\begin{array}{l}\mathrm{A} 1 \mathrm{C} 1, \mathrm{~A} 2 \mathrm{C} 2, \mathrm{~A} 3 \mathrm{C} 3, \mathrm{C} 1 \mathrm{~A} 1, \mathrm{C} 2 \mathrm{~A} 2, \mathrm{C} 3 \mathrm{~A} 3, \mathrm{~B} 1 \mathrm{D} 1, \mathrm{~B} 2 \mathrm{D} 2, \\
\text { B3D3, D1B1, D2B2, D3B3, C1E1, C2E2, C3E3, E1C1, } \\
\text { E2C2, E3C3 } \\
\text { Two-node trials }\end{array}$ & 0 & 54 & 49 \\
\hline & $\begin{array}{l}\text { A1D1, A2D2, A3D3, D1A1, D2A2, D3A3, B1E1, B2E2, } \\
\text { B3E3, E1B1, E2B2, E3B3 } \\
\text { Three-node trials }\end{array}$ & 0 & 36 & 34 \\
\hline & $\mathrm{A} 1 \mathrm{E} 1, \mathrm{~A} 2 \mathrm{E} 2, \mathrm{~A} 3 \mathrm{E} 3, \mathrm{E} 1 \mathrm{~A} 1, \mathrm{E} 2 \mathrm{~A} 2, \mathrm{E} 3 \mathrm{~A} 3$ & 0 & 18 & 17 \\
\hline
\end{tabular}

The table shows the sequences of training and the test phase, the different trial types, probabilities of programmed consequences, minimum numbers of trials, and training and test criteria during the conditional discrimination task. The first stimuli presented under trial types represent the samples and the second are the correct comparisons which were always presented together with two other incorrect comparisons

comparisons that were consistent with the experimenterdefined classes on at least $90 \%$ of the trials in one of the test blocks. Immediate emergence of the classes was said to have occurred when the criterion was reached in the first test block. Thus, the second test block measured the maintenance of the classes. Delayed emergence of the classes was documented when the criterion was not reached in the first test block but was then reached in the second test block. Failure of class formation was documented if submastery performances were obtained in both test blocks.

Simple discrimination training Before attempting to form equivalence classes, the SIM + SUCC, SIM-only, and SUCC-only groups were given some forms of discrimination training, dependent on their corresponding groups.
Participants in the SIM + SUCC group had both simultaneous and successive discrimination training, whereas the SIM-only and SUCC-only groups had simultaneous discrimination training only and successive discrimination training only, respectively.

The simultaneous discrimination training was designed with the help of a software program acquired from the University of North Texas. In the training, the $\mathrm{C}$ abstract stimuli were discriminated from other abstract stimuli $(\mathrm{X}-\mathrm{Z}$ in the bottom panel of Fig. 1) by simultaneously presenting the $\mathrm{C}$ stimuli together with the others from which they should be discriminated. The training was done in four phases, and through the phases, participants were taught to select $\mathrm{C}$ (as $\mathrm{S}^{\mathrm{D}} \mathrm{s}$ ) instead of $\mathrm{X}, \mathrm{Y}$, and $\mathrm{Z}$ (all functioning as $\mathrm{S}^{\Delta} \mathrm{s}$ ). Each response also produced a programmed consequence, either 
"correct" for the selection of the $\mathrm{C}$ stimulus or a blank screen for the selection of any other stimulus.

During Phase 1, a block of 30 trials was presented, each trial containing one of the following pairs of stimuli: $\mathrm{C} 1$ and $\mathrm{X} 1, \mathrm{C} 2$ and $\mathrm{X} 2$, or $\mathrm{C} 3$ and $\mathrm{X} 3$. Across trials, the location of the stimuli was either on the left or right of the screen and was presented in a randomized sequence. The block was repeated until ten consecutive correct responses (the selection of the $\mathrm{C}$ stimulus) have been made. Phases 2 and 3 were implemented using the same procedures, but with the use of the Y1, Y2, and $\mathrm{Y} 3$ stimuli in Phase 2, and Z1, Z2, and Z3 stimuli in Phase 3 in place of the three $X$ stimuli. Phase 4 contained a mix of all the trials from the first three phases. $\mathrm{C} 1$ was paired with $\mathrm{X} 1, \mathrm{Y} 1$, and $\mathrm{Z} 1 ; \mathrm{C} 2$ with $\mathrm{X} 2$, $\mathrm{Y} 2$, and $\mathrm{Z} 2$, and $\mathrm{C} 3$ with $\mathrm{X} 3, \mathrm{Y} 3$, and $\mathrm{Z} 3$. Ten consecutive correct responses out of the 30 trials presented were required as the mastery criterion. Phase 4 was conducted to assess the maintenance of the discrimination between the $\mathrm{C}$ stimuli and other stimuli. In each of the four phases, if the 30 trials elapsed without the participant making ten consecutive correct responses, the phase was reintroduced until the criterion was met.

After Phase 4, a test phase was introduced, with the presentation of the following pairs of stimuli: $\mathrm{C} 1$ versus $\mathrm{P} 1, \mathrm{R} 1$, or $\mathrm{S} 1$; $\mathrm{C} 2$ versus $\mathrm{P} 2, \mathrm{R} 2$, or $\mathrm{S} 2$; and $\mathrm{C} 3$ versus $\mathrm{P} 3$, $\mathrm{R} 3$, or $\mathrm{S} 3$, in a two-choice simultaneous discrimination task without the programmed consequences. All trial types were randomly presented in 30 trials, and ten consecutive correct responses were required to master the discrimination.

In the successive discrimination training, a three-ply multiple schedule was used to establish discriminations among the three $\mathrm{C}$ stimuli. When the $\mathrm{C} 1$ stimulus was presented on the screen, left-clicking on it three times (FR-3) and pressing the END button on the keyboard was followed by a "correct" message on the screen. Completion of FR-6 and FR-9 before pressing the END button occasioned "correct" programmed consequences in the presence of the $\mathrm{C} 2$ and $\mathrm{C} 3$ stimuli, respectively. Any other number of responses apart from the experimentally defined ones followed by the END button was followed with the presentation of the programmed consequence word "wrong" on the screen. Ten consecutive correct trials defined mastery and completed this phase of discrimination training.

\section{Results}

Acquisition and maintenance of the baseline relations

Median trials to acquisition were used to summarize baseline acquisition, because a few participants in some groups required many more trials than the rest in a group. As is shown in Fig. 2, the median speeds of acquiring the baseline relations were

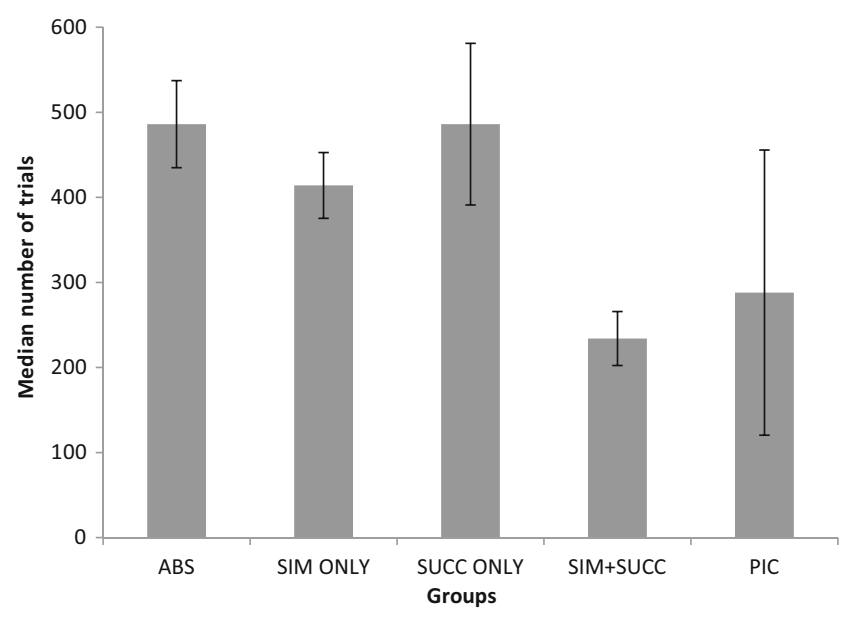

Fig. 2 Median trials needed to acquire the baseline relations in the groups. Error bars represent standard errors, $n=10$ per group

similar for participants in the SIM + SUCC and PIC groups. In addition, the baseline acquisition speeds were similar for participants in the ABS, SIM-only, and SUCC-only groups. When comparing these to clusters of groups, the speed of acquiring the baseline relations was faster for the SIM + SUCC and PIC cluster than for the ABS, SIM-only, and SUCC-only cluster; specifically, the participants in the PIC and SIM + SUCC cluster required a median of 270 trials to acquire the baseline relations, whereas the participants in the other cluster required a median of 432 trials to acquire the baseline relations. A $U$ test confirmed that the speed of acquisition of the baseline relations was faster for the PIC and SIM + SUCC groups, on the one hand, than for the ABS, SIM-only, and SUCC-only groups, on the other, $U=$ $-3.86, p<.05, r=.55$.

Across all groups, the 14 participants who formed classes required a median of 270 trials to acquire the baseline relations, as compared to 396 trials for those who did not form classes. A $U$ test showed a significant difference between those who formed equivalence classes and those who did not form classes, in terms of their speed of acquiring the baseline relations, $U=-5.292, p<.05, r=.75$.

In the absence of errors, the maintenance phase would be completed in 144 trials. Of the 50 participants in the experiment, 35 completed the maintenance phase in the minimum number of trials. The remaining 15 participants made a few errors and eventually showed the maintenance of the baseline relations in the absence of programmed consequences. The maintenance of the baseline relations was not influenced by experimental group.

Immediate, delayed, and overall emergence of equivalence classes

Table 2 shows the accuracy of responding in each test block for each participant in each group. When viewed across all 
Table 2 Accuracy of selecting class-indicative comparison selections for each participant in the two test blocks

\begin{tabular}{|c|c|c|c|c|}
\hline Group & Participant & $\begin{array}{l}\% \text { Correct in } \\
\text { Test Block } 1\end{array}$ & $\begin{array}{l}\% \text { Correct in } \\
\text { Test Block } 2\end{array}$ & $\mathrm{ECF}$ \\
\hline \multirow[t]{10}{*}{ ABS } & 4388 & 76 & 93 & YES \\
\hline & 4419 & 70 & 71 & NO \\
\hline & 4407 & 83 & 68 & NO \\
\hline & 4390 & 52 & 57 & NO \\
\hline & 4418 & 46 & 51 & NO \\
\hline & 4387 & 49 & 48 & NO \\
\hline & 4397 & 46 & 46 & NO \\
\hline & 4416 & 44 & 43 & NO \\
\hline & 4399 & 57 & 40 & NO \\
\hline & 4385 & 56 & 32 & NO \\
\hline \multirow[t]{10}{*}{ PIC } & 4411 & 97 & 100 & YES \\
\hline & 4410 & 92 & 100 & YES \\
\hline & 4380 & 86 & 100 & YES \\
\hline & 4392 & 96 & 99 & YES \\
\hline & 4405 & 87 & 99 & YES \\
\hline & 4414 & 99 & 98 & YES \\
\hline & 4384 & 99 & 98 & YES \\
\hline & 4413 & 72 & 98 & YES \\
\hline & 4395 & 70 & 73 & NO \\
\hline & 4400 & 64 & 63 & $\mathrm{NO}$ \\
\hline \multirow[t]{10}{*}{ SIM+SUCC } & 4372 & 98 & 100 & YES \\
\hline & 4377 & 82 & 100 & YES \\
\hline & 4374 & 98 & 99 & YES \\
\hline & 4373 & 99 & 98 & YES \\
\hline & 4375 & 93 & 98 & YES \\
\hline & 4379 & 86 & 93 & YES \\
\hline & 4376 & 63 & 90 & YES \\
\hline & 4371 & 61 & 86 & NO \\
\hline & 4370 & 68 & 68 & NO \\
\hline & 4378 & 54 & 54 & NO \\
\hline \multirow[t]{10}{*}{ SIM ONLY } & 4415 & 80 & 96 & YES \\
\hline & 4424 & 70 & 84 & NO \\
\hline & 4404 & 51 & 61 & NO \\
\hline & 4402 & 60 & 48 & NO \\
\hline & 4381 & 52 & 48 & NO \\
\hline & 4401 & 53 & 46 & NO \\
\hline & 4421 & 53 & 46 & NO \\
\hline & 4394 & 48 & 43 & NO \\
\hline & 4393 & 41 & 41 & $\mathrm{NO}$ \\
\hline & 4420 & 46 & 39 & $\mathrm{NO}$ \\
\hline \multirow[t]{7}{*}{ SUCC ONLY } & 4389 & 96 & 99 & YES \\
\hline & 4417 & 93 & 97 & YES \\
\hline & 4396 & 78 & 97 & YES \\
\hline & 4391 & 67 & 71 & NO \\
\hline & 4412 & 60 & 62 & NO \\
\hline & 4383 & 57 & 61 & $\mathrm{NO}$ \\
\hline & 4422 & 64 & 54 & NO \\
\hline
\end{tabular}

Table 2 (continued)

\begin{tabular}{lllll}
\hline Group & Participant & $\begin{array}{c}\text { \% Correct in } \\
\text { Test Block 1 }\end{array}$ & $\begin{array}{l}\text { \% Correct in } \\
\text { Test Block 2 }\end{array}$ & ECF \\
\hline 4409 & 57 & 47 & NO \\
4386 & 40 & 37 & NO \\
4408 & 43 & 36 & NO \\
\hline
\end{tabular}

groups, 11 of the participants showed the mastery level of class-consistent responding in both test blocks. These performances indicated the immediate emergence of the classes and their maintenance with test repetition. Nine other participants showed an absence of class-indicative performance in the first test block and then mastery in the second test block. These participants, then, showed delayed emergence of the equivalence classes.

Some of the participants who showed delayed emergence of the equivalence classes $(4380,4405,4377,4379$, and 4415) had percentages of correct responses very close to mastery levels in the first test block. The remaining 30 participants showed submastery levels of responding in both test blocks. Even with test repetition, all of these participants failed to form the experimenter-defined equivalence classes.

Effects of prior discrimination training on equivalence class formation

This experiment was designed to assess the effects of prior discrimination training on equivalence class formation, regardless of the rate of class emergence. Thus, we evaluated the effects of pre-class-formation discrimination training on the likelihood of class formation by combining yields, whether immediate or delayed, which we referred to as the overall yield. These data are illustrated in Fig. 3. Each significant outcome is also indicated by an asterisk at the end of a sentence or relevant phrase in the following discussion. The obtained probabilities associated with chi-square tests for the different comparative outcomes are listed in Table 3.

When no meaningful stimulus was included as a class member (i.e., the ABS group), only $10 \%$ of participants formed classes. The establishment of a simultaneous discriminative function produced the same yield. Thus, the prior acquisition of a simultaneous discriminative function did not enhance the subsequent probability of equivalence class formation.

In contrast, relative to the ABS yield, the establishment of a successive discriminative function produced a $20 \%$ increment in the likelihood of equivalence class formation. On the basis of visual inspection, then, the enhancement of class formation was influenced more by the prior establishment of a successive than of a simultaneous discrimination function. 


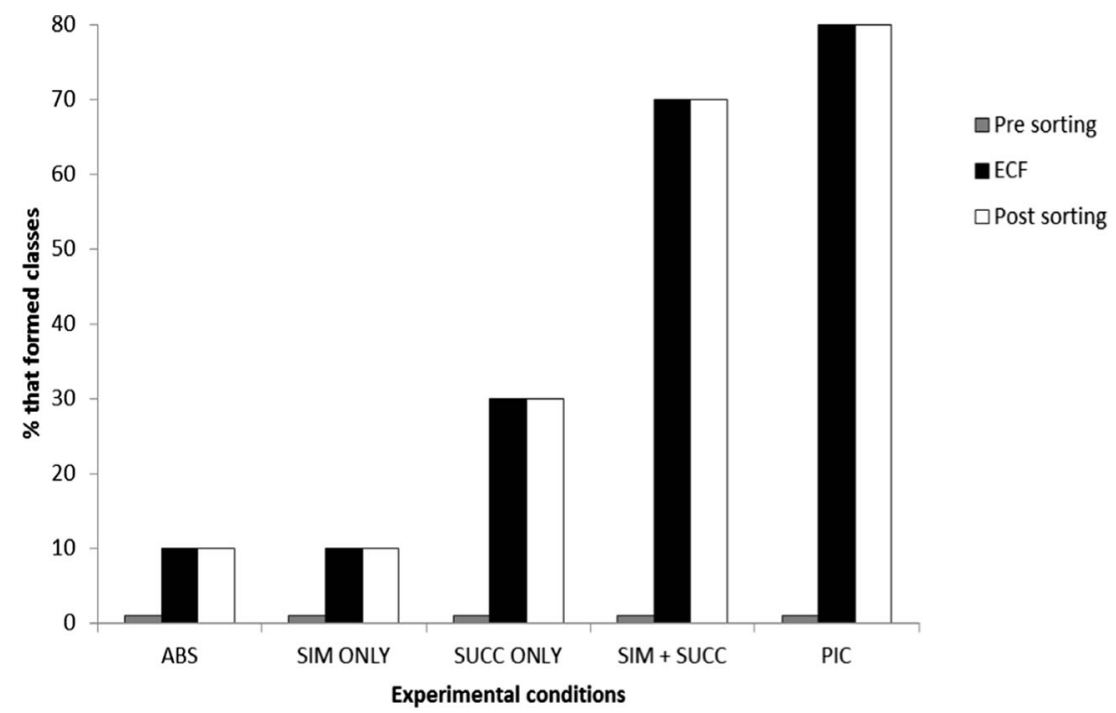

Fig. 3 Data for pre- and post-class-formation sorting tests, as well as those who formed equivalence classes in the derived relations test used to document equivalence class formation. $\mathrm{ECF}=$ equivalence class formation, $\mathrm{ABS}=$ abstract stimuli, SIM ONLY = simultaneous discrimination

Relative to the ABS yield, the combined establishment of successive and simultaneous discriminative functions produced a $60 \%$ increment in the likelihood of equivalence class formation $(70 \% \text { vs. } 10 \%)^{*}$. Furthermore, the $70 \%$ yield produced by the prior establishment of simultaneous and successive discriminative functions was greater than the $40 \%$ yield produced by summing the yields obtained after simultaneous discrimination training alone $(10 \%)$ and successive discrimination training alone $(30 \%)^{*}$. Thus, the effect of acquiring both discriminative functions together enhanced equivalence class formation in a synergistic manner.

When the PIC condition was used as a reference, class formation was much more likely to occur than when the class consisted of abstract stimuli only (ABS)*, or when the to-beformed class contained an abstract $\mathrm{C}$ stimulus that had previously acquired through a simultaneous discriminative function alone (SIM)* or a successive discriminative function alone (SUCC)*. Finally, very similar yields were produced

Table $3 P$ values for chi-square tests associated with the various group comparisons

\begin{tabular}{|c|c|c|c|c|c|}
\hline & ABS & SIM & SUCC & $\mathrm{SIM}+\mathrm{SUCC}$ & PIC \\
\hline ABS & & .234 & & .006 & .002 \\
\hline SIM & & & & .006 & .002 \\
\hline SUCC & .132 & & & .074 & .025 \\
\hline $\mathrm{SIM}+\mathrm{SUCC}$ & & & & & .606 \\
\hline SIM\&SUCC & & & & .007 & \\
\hline
\end{tabular}

Statistically significant outcomes in the table (valued less than .05) are displayed in bold font and correspond to the asterisks $(*)$ included in the discussion of Fig. 3 in the text. The SIM\&SUCC row refers to the sum of the yields produced by SIM alone and SUCC alone training only, SUCC ONLY = successive discrimination training only, $\mathrm{SIM}+\mathrm{SUCC}=$ simultaneous + successive discrimination training, and $\mathrm{PIC}=$ picture stimuli

when the classes contained a meaningful stimulus (PIC) or an abstract $\mathrm{C}$ stimulus that had acquired both simultaneous and successive discriminative functions prior to class formation. Thus, the class-enhancing effects of including a meaningful stimulus in an equivalence class was matched by the inclusion of an abstract stimulus as a class member, as long as it had acquired simultaneous and successive discriminative functions prior to class formation.

\section{Card sorting}

The data obtained from the pre- and post-class-formation sorting tests for all participants are presented on the left and right sides, respectively, of Table 4. Before class formation training, none of the participants sorted the stimuli into any of the experimenter-defined classes. Instead, they produced subject-defined classes that contained mixes of stimuli different from those in the experimenter-defined classes, in sets that contained from two to five stimuli.

After exposure to the simultaneous protocol, 20 of the 50 participants across all of the conditions sorted the stimuli into the three experimenter-defined classes. The remaining participants sorted the stimuli into clusters that did not correspond to the experimenter-defined classes.

Figure 3 includes the outcomes of sorting, along with the results of the emergent-relations test data used to document class formation. After administration of the derived-relations tests, all of the participants who formed classes during the derived-relations tests also showed maintenance of those classes during the sorting test. Also, participants who did not form classes also did not sort the stimuli into the experimenterdefined classes. Thus, there was $100 \%$ concordance of the 
Table 4 Pre- and post-class-formation card sorting for all participants

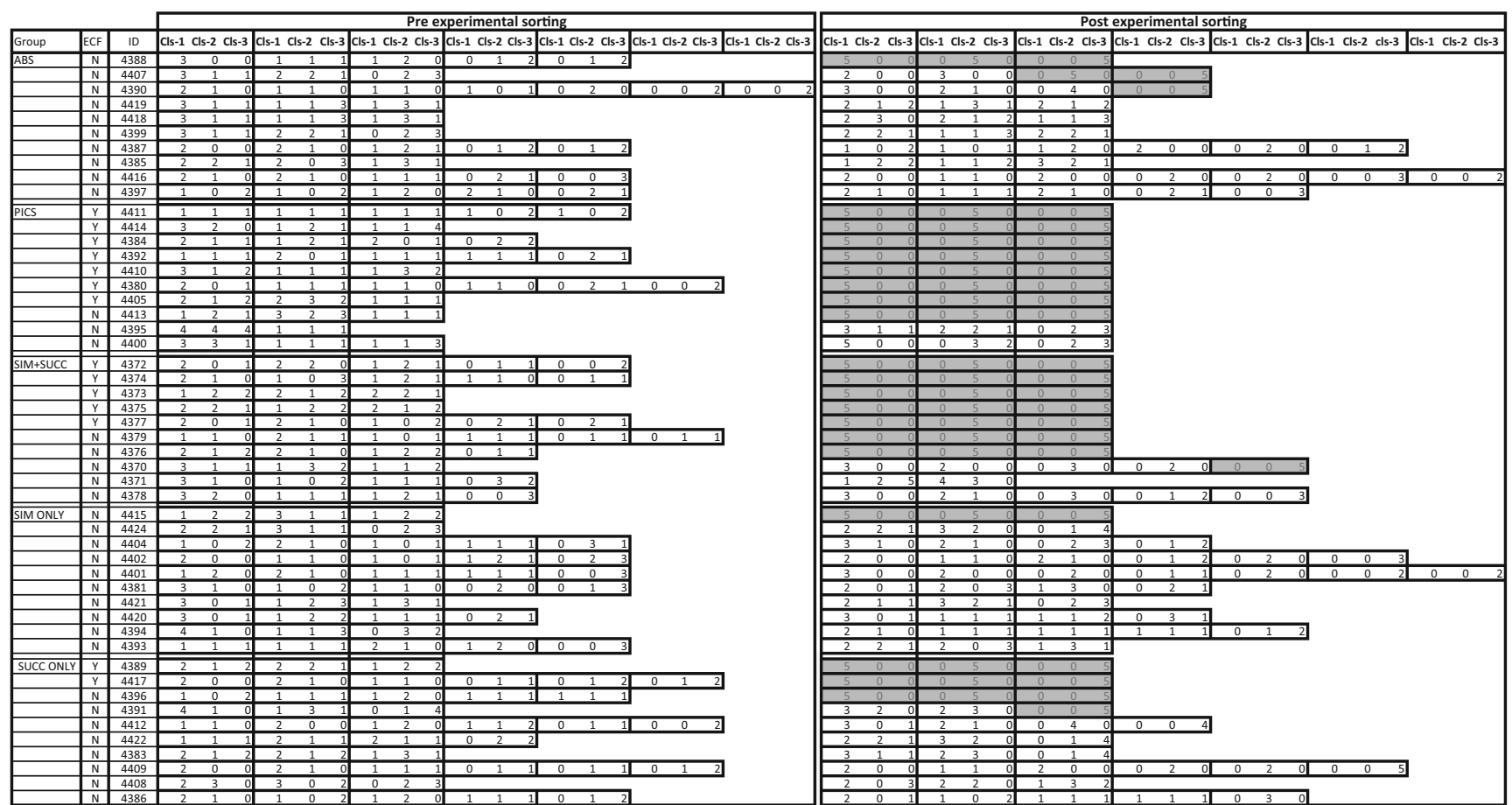

Rows are grouped by experimental groups, then by success $(\mathrm{Y})$ or failure $(\mathrm{N})$ at equivalence class formation. Each row is divided into boxes, each of which contains information for one of the subject-defined classes. All boxes have three cells, each of which contains information for the experimentally defined Classes 1,2, and 3, respectively. The entries in each cell indicate the number of stimuli in each experimenter-defined class that the participant assigned to one subject-defined class. Thus, the number " 500 " in the three cells in a box would indicate that the five stimuli in Class 1 were clustered into a subject-defined class, whereas an entry of 302 would indicate a five-member participant-defined class that contained three stimuli from Class 1 , zero stimuli from Class 2, and two stimuli from Class 3. The boxes with gray backgrounds indicate sorting that corresponds to an experimenter-defined class

performances produced by the class formation test administered using MTS-based derived-relations trials and the maintenance tests administered using a card-sorting format.

\section{Discussion}

In the present experiment, we investigated the differential effects of pretraining with simultaneous and/or successive discriminations on the subsequent formation of three-node, five-member classes for which both training and testing were administered under the simultaneous protocol. (1) Very few formed classes when the middle nodes as well as the other class members were abstract and meaningless stimuli (ABS). (2) Most participants did form classes when the middle node in the class was a familiar picture (PIC) and the other class members were abstract stimuli. (3) Abstract stimuli that had acquired successive and simultaneous discriminative functions (SIM + SUCC) prior to class formation facilitated class formation that was similar to that found when the middle node was a familiar picture. (4) When either the successive or the simultaneous discriminative function was acquired by an abstract stimulus alone, successive discrimination training
(SUCC-only) produced higher yields than did simultaneous discrimination training (SIM-only). (5) The sum of the two yields produced by the separate (simultaneous and successive) discriminations was not as large as performance produced by the establishment of both discriminative functions. (6) Finally, a sorting test confirmed the formation of these classes by all of the participants who formed classes during the derivedrelations test.

In general, Results 1, 2, 3, and 6 above replicated the findings reported by Fields et al. (2012) and by Travis et al. (2014). The new findings of the present experiment are those mentioned in Results 4 and 5, each of which will be considered below.

Synergistic effects of simultaneous and successive discriminative functions

Travis et al. (2014) explored the effects of pre-class-formation establishment of SUCC-only and SIM + SUCC discrimination training on equivalence class formation and found that class formation was enhanced more by the prior formation of both types of discriminations than by the prior formation of successive discriminations alone. Furthermore, by the subtraction of one yield from the other, he predicted that the 
formation of SUCC discriminations alone should enhance class formation more than the formation of simultaneous discriminations would. This inference was based on a subtractive theoretical analysis rather than on empirical data. The results of the present experiment confirmed that prediction.

Travis et al. (2014) also speculated about the possible additive effects of SIM + SUCC training, but did not draw any definitive conclusions. The present experiment has provided a direct answer on this issue. Specifically, the increase in yield produced by the prior establishment of both successive and simultaneous discriminations was greater than that produced by summing the class-enhancing effects produced by prior establishment of successive discriminations alone and simultaneous discriminations alone. These results, then, show that the establishment of simultaneous and successive discriminations acts in a synergistic manner, so that the establishment of one enhances the effect of the other pre-class-formation training effect: With respect to the class-enhancing effects of the two discriminative functions, the whole appears to be greater than the sum of the parts.

\section{Differential effects of simultaneous and successive discriminations}

The acquisition of C-based simultaneous discriminations produced no class enhancement effect. In contrast, the acquisition of the C-based successive discriminations produced a modest enhancement of class formation. The following is a speculative analysis of a factor or mechanism that might account for the superior enhancement effect produced by the pre-classformation establishment of successive versus simultaneous discriminations.

The successive discrimination procedure used in the present experiment required the emission of topographically different responses in the presence of each $\mathrm{C}$ stimulus: that is, FR3, FR6, and FR9 responses for the $\mathrm{C} 1, \mathrm{C} 2$, and $\mathrm{C} 3$ stimuli, respectively. These responses provided a basis for response coding of the stimuli (Urcuioli, 2013) and might have prompted the emergence of a generalized stimulus-coding repertoire. Such a repertoire would then generalize to the emergent-relations tests, in which the participants would code the sample stimuli in the derived-relations test trials; facilitate the selection of a comparison from the same class; and thereby increase the likelihood of class formation.

In contrast, during the formation of a simultaneous discrimination, both stimuli are presented concurrently. The correct response involved the selection of the $\mathrm{S}^{\mathrm{D}}$. Since the same selection-based response topography was required even if the $\mathrm{S}^{\Delta}$ was chosen, the contingencies of reinforcement were less likely to induce the formation of a generalized coding repertoire. Since it is unlikely that coding behavior was induced, that repertoire was less likely to generalize to and facilitate the solution of the derived-relations probes, thereby enhancing the likelihood of equivalence class formation. On the other hand, it is not plausible to argue that nothing was acquired during the formation of the simultaneous discriminations, because of the synergistic effects of training both the simultaneous and successive discriminations. This admittedly speculative analysis would have to be evaluated with additional research that sought to monitor the presence of coding behavior during the formation of the successive and simultaneous discriminations and during the emergent-relations tests.

Sidman (1994) argued that, since the two stimuli are presented at the same time in the simultaneous discrimination procedure, the relational characteristics of the stimuli might be easier for the participant to notice than when the stimuli are presented separately. The present study, however, showed contradictory findings, with a history of successive discrimination training being superior, in terms of the likelihood of equivalence class formation, to simultaneous discrimination training.

If simultaneous discrimination is easier than successive discrimination (Sidman, 1994), and if both are important prerequisites for the establishment of conditional relations (Saunders \& Green, 1999), then a history of successive discrimination training, which is a more difficult repertoire, is far more advantageous to have than a history of simultaneous discrimination training. Thus, having been trained with the more difficult of the prerequisites for forming conditional relations is more favorable than the easier prerequisite. However, since the simultaneous discrimination could have been established by stimulus control based on either selection or rejection, it is possible that most of the participants learned to respond away from the $\mathrm{S}^{\Delta}$, or by rejection, which is a weaker form of stimulus control and one that is bound to lead to failure in the subsequent establishment of conditional relations. The general test for simultaneous discriminations with novel $\mathrm{S}^{\Delta} \mathrm{S}$, however, suggests that that is not the case. The fact that we found a synergistic effect of training SIM + SUCC provides further evidence that control of behavior had been acquired by the "accept" relation in simultaneous discriminations.

\section{Potential effects of near misses}

When the data in Table 2 are considered, two participants (4371 and 4424) did not form classes but responded with increasing accuracy in the second test block relative to the first, with performances that approached the criterion used to define class formation. For these participants, the data suggest eventual class formation with additional repetitions of the test block. Were this to have occurred, it would have produced slight increases in the yields obtained in the SUCC + SIM and SIM-only groups. These increases, however, would not have changed the conclusions that we have drawn from the data as analyzed in the present experiment. 
Stimulus control functions of meaningful stimuli

The meaningfulness established by pretraining of otherwise abstract stimuli has been defined by their denotative properties, their connotative properties, and more recently by the presumed discriminative properties exerted by these stimuli (e.g., Fields et al., 2012; Tyndall, Roche, \& James, 2004). Specifically, meaningful stimuli can serve as $\mathrm{S}^{\mathrm{D}} \mathrm{s}$ in successive and simultaneous discriminations, as sample and/or comparison stimuli in identity or arbitrary conditional discriminations, and/or as members of perceptual categories or equivalence classes (Fields et al., 2012; Travis et al., 2014). Many experiments have shown that the inclusion of at least one meaningful stimulus in a set of otherwise meaningless stimuli will facilitate the formation of an equivalence class. Thus, the class-enhancing effects of a meaningful stimulus on equivalence class formation might be accounted for in part by some of its inferred acquired stimulus control functions. The results of research presented by Tyndall et al. (2004), Leslie et al. (1993), Fields et al. (2012), and Travis et al. (2014) support such a perspective. The present research also supports and extends this perspective, suggesting that many behavioral stimulus control functions are presumably acquired by meaningful stimuli, which can account for their ability to enhance the likelihood of forming equivalence classes. Additional research will be needed to determine whether other, unexplored stimulus control functions presumably served by meaningful stimuli also contribute to their ability to enhance equivalence class formation.

As we noted above, these experiments were conducted using the simultaneous protocol, a procedure that minimizes the likelihood of class formation. Regardless of the implications of the present experiment about the enhancing effects of meaningful stimuli on class formation, the results of the present experiment add to the growing number of variables that enhance the formation of equivalence classes when training and testing are conducted under the simultaneous protocol. The application of these procedures may facilitate learning under such conditions.

\section{Card sorting}

None of the participants sorted the stimuli into experimenterdefined classes prior to the experiment. However, after exposure to training and testing, regardless of condition or the speed of emergence, participants who formed classes also sorted the stimuli into the experimenter-defined classes, and those who did not form classes also did not sort in accord with equivalence. This shows that performance on card sorting was influenced by the contingencies set in the training and testing for derived relations. These data also show that class-based behavior generalized between two trial formats: matching-tosample trials during class formation, and sorting during post- class-formation testing. Finally, the post-class-formation sorting test documented the maintenance of the classes that had been formed under the simultaneous protocol. Similar findings have been reported by Fields, Arntzen, and Moksness (2014).

\section{Summary}

The inclusion of a meaningful stimulus in a set of meaningless stimuli enhanced the probability of forming equivalence classes with that set of stimuli. This effect was also produced by the establishment of a simple simultaneous discriminative function in combination with a simple successive discriminative function for one of the meaningless stimuli in a to-beformed equivalence class. The magnitude of class enhancement was influenced more by the acquisition of a successive discriminative function alone than by the acquisition of a simultaneous discriminative function alone. The acquisition of both functions for a meaningless stimulus had a greater effect than did the sum of the acquisitions of each of the separate functions. Finally, a post-class-formation sorting test documented the maintenance of the classes that had been formed under the simultaneous protocol. These results, then, imply that some portion of the class-enhancing effects of meaningful stimuli can be accounted for by the successive and discriminative functions presumably served by meaningful stimuli.

Author note There are no conflicts of interest to declare concerning the three authors.

\section{References}

Arntzen, E. (2004). Probability of equivalence formation: Familiar stimuli and training sequence. Psychological Record, 54(2), 7:275-291. Retrieved from http://opensiuc.lib.siu.edu/tpr/vol54/iss2/7/

Arntzen, E., \& Lian, T. (2010). Trained and derived relations with pictures as nodes. Psychological Record, 60(4), 8:659-677. Retrieved from http://opensiuc.lib.siu.edu/tpr/vol60/iss4/8/

Fields, L., \& Verhave, T. (1987). The structure of equivalence classes. Journal of the Experimental Analysis of Behavior, 48, 317-332. doi: 10.1901/jeab. 1987.48-317

Fields, L., Arntzen, E., Nartey, R. K., \& Eilifsen, C. (2012). Effects of a meaningful, a discriminative, and a meaningless stimulus on equivalence class formation. Journal of the Experimental Analysis of Behavior, 97, 163-181. doi:10.1901/jeab. 2012.97-163

Fields, L., Arntzen, E., \& Moksness, M. (2014). Stimulus sorting: A quick and sensitive index of equivalence class formation. Psychological Record, 64, 487-498. doi:10.1007/s40732-0140034-y

Leslie, J. C., Tierney, K. J., Robinson, C. P., Keenan, M., Watt, A., \& Barnes, D. (1993). Differences between clinically anxious and nonanxious subjects in a stimulus equivalence training task involving threat words. Psychological Record, 43, 153-161. 
Mcllvane, W. J. (2013). Simple and complex discrimination learnimg. In G. Madden, W. V. Dube, T. D. Hackenberg, G. P. Hanley, \& K. A. Lattal (Eds.), APA handbook of behavior analysis (Vol. 1, pp. 129163). Washington, DC: American Psychological Association.

Nartey, R. K., Arntzen, E., \& Fields, L. (2014). Two discriminative functions of meaningful stimuli that enhance equivalence class formation. The Psychological Record. doi:10.1007/s40732-014-0072-5

Saunders, R. R., \& Green, G. (1999). A discrimination analysis of training-structure effects on stimulus equivalence outcomes. Journal of the Experimental Analysis of Behavior, 72, 117-137. doi:10.1901/jeab. 1999.72-117

Sidman, M. (1994). Equivalence relations and behavior: A research story. Boston: Authors Cooperative.

Sidman, M., \& Tailby, W. (1982). Conditional discrimination vs. matching to sample: An expansion of the testing paradigm.
Journal of the Experimental Analysis of Behavior, 37, 5-22. doi: 10.1901/jeab.1982.37-5

Travis, R. W., Fields, L., \& Arntzen, E. (2014). Discriminative functions and over-training as class-enhancing determinants of meaningful stimuli. Journal of the Experimental Analysis of Behavior. doi:10. 1002/jeab.91

Tyndall, I. T., Roche, B., \& James, J. E. (2004). The relation between stimulus function and equivalence class formation. Journal of the Experimental Analysis of Behavior, 81, 257-266. doi:10.1901/jeab. 2004.81-257

Urcuioli, P. J. (2013). Stimulus control and stimulus class formation. In G. Madden, W. V. Dube, T. D. Hackenberg, G. P. Hanley, \& K. A. Lattal (Eds.), APA handbook of behavior analysis (Vol. 1, pp. 361-386). Washington, DC: American Psychological Association. 